\title{
Uterus Bicorne Cervical United: A Case of Pregnancy after Uterine Plasty (Review of the Literature)
}

\author{
L. Soumare ${ }^{1}$, O. Sacko1, S. Diallo², M. Sissoko' ${ }^{1}$, S. Koumare1, M. Camara1,

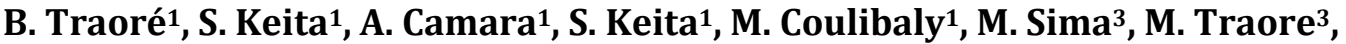 \\ M. D. Soumare ${ }^{3}$, A. Koita', D. Traore' ${ }^{2}$, Z. Z. Sanogo' ${ }^{1}$
}

${ }^{1}$ Service of Surgery A of CHU POINT G, Bamako, Mali

${ }^{2}$ Service of Surgery B of CHU POINT G, Bamako, Mali

${ }^{3}$ Service of Gynecology of CHU POINT G, Bamako, Mali

Email:^drsoumare@gmail.com

How to cite this paper: Soumare, L., Sacko, O., Diallo, S., Sissoko, M., Koumare, S., Camara, M., Traoré, B., Keita, S., Camara, A., Keita, S., Coulibaly, M., Sima, M., Traore, M., Soumare, M.D., Koita, A., Traore, D. and Sanogo, Z.Z. (2019) Uterus Bicorne Cervical United: A Case of Pregnancy after Uterine Plasty (Review of the Literature). Surgical Science, 10, 413-419. https://doi.org/10.4236/ss.2019.1012046

Received: September 4, 2019

Accepted: December 23, 2019

Published: December 26, 2019

Copyright $\odot 2019$ by author(s) and Scientific Research Publishing Inc. This work is licensed under the Creative Commons Attribution International License (CC BY 4.0).

http://creativecommons.org/licenses/by/4.0/

\section{(c) (i) Open Access}

\begin{abstract}
The uterus bicorne is a uterine malformation whose pathophysiology is known but the etiology remains obscure. Few studies have been interested in this subject. This is the clinical observation of Mrs. X 26-year-old housewife nulliparous who consulted for repeated late spontaneous abortions. The clinical examination was normal. The ultrasound revealed a double uterine cavity in $\mathrm{Y}$, in favor of a unicervical bicorne uterus. At hysterosalpingography, the tubes were permeable and the uterus doubled with a single cervix. The treatment consisted of a uterine plasty. The postoperative course was simple. The patient gave birth to a newborn male 20 months after surgery.
\end{abstract}

\section{Keywords}

Uterus Bicornis Unicollis, Malformation, Uterine Plasty, Pregnancy

\section{Introduction}

The real incidence of uterine malformations is difficult to assess in the literature [1]. The bicornous uterus is a uterine malformation. The bicorne uterus can be one cervical or two cervicals. When he is two or bi cervical he is called by the Anglo Saxon uterus didelphe. The pathophysiology of these malformations is known but the etiologies remain obscure and few studies have been interested. Its frequency in uterine malformations varies from $39 \%$ to $45 \%$. In fertile women there is $41 \%$ of bicorn uterus while in infertile women there is $25 \%$ of bicornous 
uterus. The bicornous uterus may be subject to recurrent miscarriage. The restoration of a normal uterinecavity is the basis of their treatment [2]. It may require uterine plasty. We report here a case of pregnancy after uterine plasty of a cervical united bicorn uterus.

\section{Clinical Observation}

Mrs X housewife of nullipare 26 years consulted for repeated late spontaneous abortions. In her antecedents we noted 7 spontaneous late miscarriages occurring between the $4^{\text {th }}$ and the $6^{\text {th }}$ month of pregnancy. These abortions occur in an apyretic context without obstetrical trauma. On examination there was no pain, no dysmenorrhea, no cycle disorder and no urinary disorder. We did not note abnormalities in morphotype, development of secondary sexual characteristics, hair growth or breast development. On speculum examination we observed a single cervix without any particularity. The vaginal touch was normal.

The ultrasound revealed a double uterine cavity joining $\mathrm{Y}$ at the cervix, in favor of a cervical united bicorn uterus. In Hysterosalpingography, the tubes were permeable and the uterus doubled with a single cervix (Figure 1). The technical platform did not allow us to perform the karyotype.

Surgery was decided and performed. It was performed under general anesthesia with orotracheal intubation. After open coelio we proceeded to the establishment of a trocar of $10 \mathrm{~mm}$ under umbilical and two trocars of $5 \mathrm{~mm}$ in the iliac pits. At the introduction of optics we did not note any hepatitis peri or pelvic adhesions. We found a uterus bicorne united cervical whose right half was more developed than the left half. From each half of the uterus was a trunk ending in a well-bloomed flag, ovaries of normal appearance, and round ligaments in place. A pseudo ligament doubling the right broad ligament started from the base of the coecum to the posterolateral aspect of the right uterine half. We decided to convert to conventional surgery after marking the benchmarks of the plasty on the serosa of the inner edge of the two half-uteri. We performed a medial

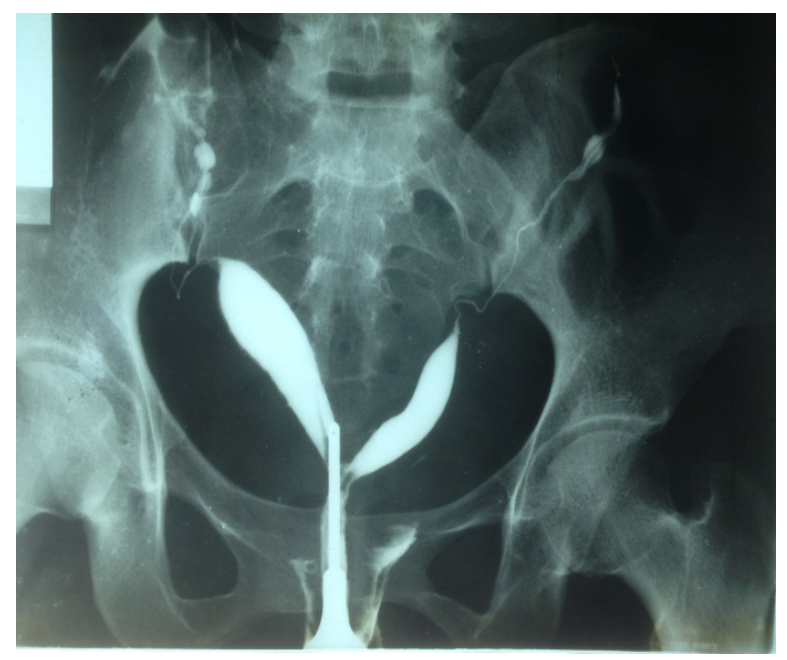

Figure 1. Preoperative hysterosalpingography. 
incision under umbilical. At the opening the carrot made from the previously made marks was removed with the electrocautery passing flush with the mucosa on both sides. The mucosa was then opened to the mono-polar section. The common cervical canal was easily catheterized with a forceps and then an indwelling catheter was placed vaginally and collected through the abdominal opening. Plasty was performed by a plane of separated points with vicryl 2/0. The balloon of the catheter is inflated in the uterine cavity at 2 CC. Peritoneal lavage with saline followed by closure of the wall in 3 planes. The postoperative course was simple. The patient returned to her home on J5 postoperative. Hysterosalpingography was performed 5 months after surgery, the uterus had a single cavity with a cervix (Figure 2). After 8 months postoperatively the patient contracted a pregnancy which aborted spontaneously at 20 weeks of amenorrhea. A second pregnancy 1 year later gave birth to a newborn male weighing $3200 \mathrm{~g}$.

\section{Comments}

Prevalence studies in the general population have biases because these malformations are often asymptomatic. The prevalence of uterine malformations found in women consulting for tubal infertility is between $0.17 \%$ and $5.6 \%$ according to the authors [3]. The prevalence varies between fertile women $3 \%$ to $3.5 \%$ and infertile women 2.3\%. ZHANG Yan at Peking University had $0.45 \%$ uterine malformations [4]. The various types of malformations are the arched uterus $7 \%$ to $15 \%$, the septal uterus $12 \%$ to $34 \%$, the uterus unicorn and pseudo unicorn $4.4 \%$ to $5 \%$, the uterus bicorne $39 \%$ to $45 \%$ and the Uterus didelphe $11 \%$. ZHANG Yan and all had $10.3 \%$ bicorn uterus.

\section{1) Organogenesis}

The bicornous uterus results from the non closure of the Müller canals between the $9^{\text {th }}$ and $13^{\text {th }}$ week of development. The Müller's canal is formed by an invagination of the coelomic epithelium on the anterolateral side of the urogenital crest.

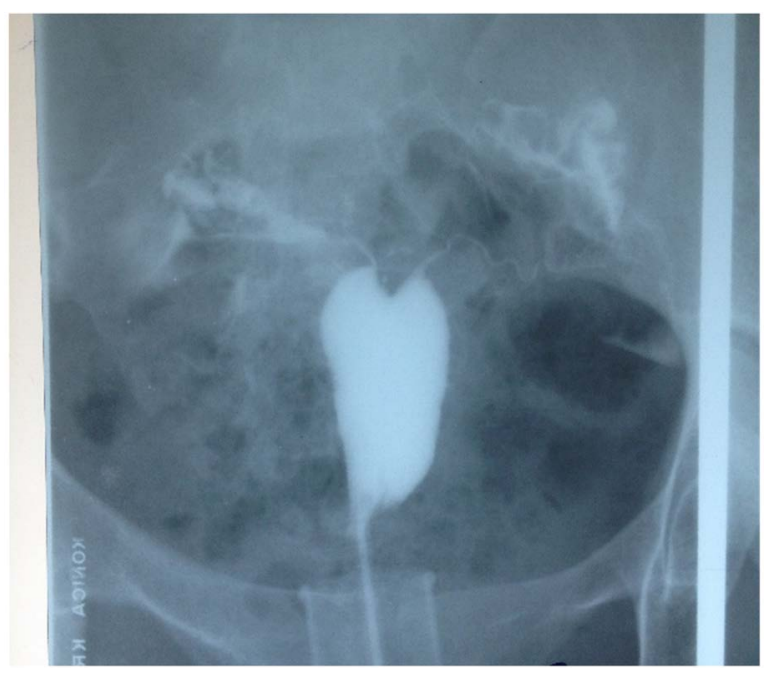

Figure 2. Postoperative hysterosalpingography. 
The appearance and progression of Müller's canals to the urogenital sinus occurs between the $6^{\text {th }}$ and $9^{\text {th }}$ weeks. The two channels are initially separated by a septum but then merge to form the urethrovaginal canal between the $10^{\text {th }}$ and $13^{\text {th }}$ weeks. The septum can persist between the 2 channels resulting in the formation of a bicorne uterus.

The karyotype of patients is normal in more than $90 \%$ of cases [3]. Karyotype abnormalities (trisomy 18, trisomy 13, monosomy X) have been found by some authors, their frequency is between $7.7 \%$ and $9.3 \%$ of cases [3]

Some authors have found cytogenetic abnormalities in women with uterine malformation, deletion of the long arms of the $\mathrm{X}$ chromosome or Robertsonian translocations of chromosomes 9 and 17 [3].

\section{2) Etiologies}

Two iatrogenic causes have been described as responsible for uterine malformations. It is on the one hand thalidomide responsible for uterus bicorne and Rokitansky syndromes, and secondly, and better known, diethylstilbestrol (Distilbent). Uterine malformations are associated with malformations of the upper urinary tract in about $30 \%$ of cases [3].

The frequency of upper genital malformations associated with cloacal malformation is estimated to be between $9 \%$ and $25 \%$ [3]. We did not find any renal or anorectal malformations in our patient.

\section{3) Clinical}

The cervical united bicorn uterus is most often asymptomatic. The discovery is often fortuitous during imaging examinations, revision, during an abdominopelvic surgery performed for another reason or during a caesarean section performed for abnormal work or presentation. It can be revealed during the assessment for spontaneous premature miscarriages repeated during the assessment of a renal malformation or during a curettage failure. The speculum examination may find a longitudinal septum or a blind pocket. The presence of 2 cervical spines requires imaging tests to look for upper genital malformation. Rarely the vaginal touch finds a hollow uterus. Diagnosis is based on imaging exams.

Hysterosalpingography remains an examination of current practice in the context of sterility tests but is never sufficient in itself for the diagnosis of uterine malformation. It gives information only on the morphology of the uterine cavity and not on the external aspect of the uterus. The specificity of hysterography used alone in the diagnosis of uterine malformations is only $17.5 \%$ to $55 \%$, $62.5 \%$ in combination with clinical examination and $90 \%$ in combination with ultrasound [3]. In the case of unicervical bicorne uterus, there is theoretically an angle greater than $90^{\circ}$ between the two horns, but hysterography alone does not allow the differential diagnosis between bicorne and septate uterus.

The ultrasound should be best performed in the second half of the cycle to benefit from the increased echogenicity of the mucosa at this time.

In cervical or cervical bicorne uteri, echography typically reveals uterine cavi- 
ties separated by a V-shaped herniation of the posterior bladder wall, which is in fact an inconstant and non-specific sign that can also be seen in uteri cloisonné [3].

According to the authors, the sensitivity of endovaginal ultrasonography is between $57 \%$ and $100 \%$ for the diagnosis of uterine malformations. Its specificity for the diagnosis of partition varies between $33 \%$ and $80 \%$, whereas for the bicorne the specificity is of $100 \%$ [3]. Three-dimensional ultrasound is particularly useful for the diagnosis of septate and bicorne uteri, as it allows the diagnosis of septate uterus in $92 \%$ of cases and of bicornous uterus in $100 \%$ of cases [3].

Ultrasound also makes it possible, during the same examination, to look for an associated malformation of the upper urinary tract, which must be routinely performed in front of any uterine malformation.

The distinction between cocked hat and cloisonné, however, remains difficult and error-prone. It is very important to study the uterine fundus well on the different sections. All malformations combined, the sensitivity of the MRI is $77 \%$ to $100 \%$, the specificity of $33 \%$ to $100 \%$ [3]. To perform the differential diagnosis between septate uterus and bicornous uterus, MRI has a sensitivity of $73 \%$, a specificity of $66 \%$ and a positive predictive value of $89 \%$ [3].

Laparoscopy is still the only diagnostic device that can confirm the external form of the uterus with certainty. In the bicorne uteri, laparoscopy allows to visualize the two horns separated by a furrow. It allows to appreciate the morphology of the ovaries and to look for associated lesions of endometriosis. Laparoscopy is an important examination in the diagnosis of uterine malformations. It is not carried out systematically and is justified only in case of infertility or associated pelvic pain or even if one considers a therapeutic act, taking into account the potential morbidity of this operative act and the clear progress of non-invasive imaging means. In our case we did a first laparoscopy before conversion to conventional surgery for septum rupture

The frequency of early spontaneous miscarriages for all malformed uteri varies from $22 \%$ to $36 \%$ of pregnancies according to the authors [3]. For ZHANG Yan the bicornous uterus is not in itself a factor of infertility [4]. For different authors, $62 \%$ of pregnancies evolve beyond 24 weeks of amenorrhea [3]. Y. Y. CHAN et al. Find that women with bicorn or unicorn uterus are more prone to miscarriage than women with normal uteri [5]. The frequency of premature deliveries is between $15 \%$ and $23 \%$ for the bicornous uteri [3].

\section{4) Design}

Term birth occurs in: $35 \%$ to $70 \%$ of the bicorns [3] [6]. Cruceyra et al. reported a case of twin pregnancy on bicornous uterus [5]. In this case each fetus evolved on both sides of the septum and the delivery was done by elective bilateral low transverse cesarean section. Y. Y. CHAN et al. Reported that abnormal fetal presentation rates are higher in the female population with bicorn or unicorn uterus than in the population of women with normal uteri [5]. The rate of ectopic pregnancies reported by the various authors is higher in the case of ute- 
rine malformation than in the general population, with an overall rate of $3 \%$ of which $2.5 \%$ for the bicornous uteri.

\section{5) Treatment}

In the case of a bi-cervical bicorne uterus with a blind hemi-vagina, the treatment usually consists of a hemi-hysterectomy associated with the correction of the vaginal malformation. In the other types of bicornous uteri, the surgical treatment of the malformation is Strassman's intervention [3] which consists of a transverse hysterotomy from one horn to the other, followed by either a disinvagination of the spur or resection of the spur. This procedure can damage the interstitial portion of the tubes. It is justified only in case of symptomatic malformation. Magendie's intervention consisted of a resection of the internal septum of each hemiorne followed by a suture of reunification of the two hemicornae. Cobellis L. reports a case of unicervical bicorne uterus operated by Jones' technique [7]. This technique involves the rupture of the septum separating the two cavities after laparotomy. This intervention resulted in pregnancy and premature delivery after 32 weeks of amenorrhea. Zhang Yan et al. find that women who have undergone hysteroscopic resection of the septum have a $75 \%$ term delivery rate and a live birth rate of about $85 \%$ [5]. Postoperatively, the uterus is scar. It is a scar performed in a context outside the obstetrical context and therefore very solid [8]. During childbirth, careful use of oxytocics and locoregional analgesia is possible [8].

\section{Conclusion}

Uterine malformations have a certain obstetrical impact. The diagnosis of these malformations is possible on ultrasound in most cases. The impact of these malformations can be limited thanks to the surgical and/or endoscopic treatment that improves the obstetric prognosis.

\section{Conflicts of Interest}

The authors declare no conflicts of interest regarding the publication of this paper.

\section{References}

[1] Alaoui, F.Z.F., Bouguern, H., Jayi, S., Squalli, N. and Melhouf, M.A. (2012) Conduite à tenir devant un utérus didelphe associé à un hémivagin borgne. Pan African Medical Journal, 13, 56.

[2] Grimbizis, G.F., Camus, M., Tarlatzis, B.C., Bontis, J.N. and Devroey, P. (2001) Clinical Implications of Uterine Malformations and Hysteroscopic Treatment Results. Human Reproduction Update, 7, 161-74. https://doi.org/10.1093/humupd/7.2.161

[3] Savey, L. and Le Tohic, A. Malformations utérines EMC gynecologie123-A-10: 1-17.

[4] Zhang, Y., Zhao, Y.-Y. and Qiao, J. (2010) Obstetric Outcome of Women with Uterine Anomalies in China. Chinese Medical Journal, 123, 418-422.

[5] Chan, Y.Y., Jayaprakasan, K., Tan, A., Thornton, J.G., Coomarasamy, A., Raine- 
Fenning, N.J. (2011) Reproductive Outcomes in Women with Congenital Uterine Anomalies: A Systematic Review. Ultrasound in Obstetrics \& Gynecology, 38, 371382. https://doi.org/10.1002/uog.10056

[6] Cobellis, L., Messalli, E.M., Pierno, G. and Cobellis, G. (2001) A Case of Atypical Bicornate Unicollis Uterus Treated with Metroplasty According to Jones. Minerva Ginecologica, 53, 289-291.

[7] Cruceyra, M., Iglesias, C., De La Calle, M., Sancha, M., Magallón, S.L. and González, A. (2011) Successful Delivery of a Twin Pregnancy in a Bicornuate Uterus (Uterus Bicornis Unicollis) by Bilateral Caesarean Section. Journal of Obstetrics and Gynaecology Canada, 33, 142-144. https://doi.org/10.1016/S1701-2163(16)34800-9

[8] Rudigoz, R.C. and Gaucherand, P. Retentissement obstétrical des malformations utérines. EMC obstétrique 5-061-A-10. 